\title{
Identified members of the Streptomyces lividans AdpA regulon involved in differentiation and secondary metabolism
}

\author{
Aurélie Guyet ${ }^{1}$, Nadia Benaroudj², Caroline Proux ${ }^{3}$, Myriam Gominet ${ }^{1}$, Jean-Yves Coppée ${ }^{3}$ and Philippe Mazodier ${ }^{1 *}$
}

\begin{abstract}
Background: AdpA is a key transcriptional regulator involved in the complex growth cycle of Streptomyces. Streptomyces are Gram-positive bacteria well-known for their production of secondary metabolites and antibiotics. Most work on AdpA has been in S. griseus, and little is known about the pathways it controls in other Streptomyces spp. We recently discovered interplay between ClpP peptidases and AdpA in S. lividans. Here, we report the identification of genes directly regulated by AdpA in S. lividans.

Results: Microarray experiments revealed that the expression of hundreds of genes was affected in a S. lividans adpA mutant during early stationary phase cultures in YEME liquid medium. We studied the expression of the $S$. lividans AdpA-regulated genes by quantitative real-time PCR analysis after various times of growth. In silico analysis revealed the presence of potential AdpA-binding sites upstream from these genes; electrophoretic mobility shift assays indicated that AdpA binds directly to their promoter regions. This work identifies new pathways directly controlled by AdpA and that are involved in S. lividans development (ramR, SLI7885 also known as hyaS and SLI6586), and primary (SLI0755-SLI0754 encoding CYP105D5 and Fdx4) or secondary (cchA, cchB, and hyaS) metabolism.

Conclusions: We characterised six S. lividans AdpA-dependent genes whose expression is directly activated by this pleiotropic regulator. Several of these genes are orthologous to bldA-dependent genes in S. coelicolor. Furthermore, in silico analysis suggests that over hundred genes may be directly activated or repressed by S. lividans AdpA, although few have been described as being part of any Streptomyces AdpA regulons. This study increases the number of known AdpA-regulated pathways in Streptomyces spp.
\end{abstract}

Keywords: Streptomyces, lividans, Microarrays, AdpA, bldA, ramR, hyaS, CYP105D5, cchB, Coelichelin

\section{Background}

Streptomycetes are Gram-positive soil bacteria that display a complex morphological and metabolic differentiation. Streptomyces develop branched hyphae that expand by tip extension to form a vegetative mycelium meshwork. In response to as yet unidentified signals and to nutritient depletion, aerial branches emerge from the surface of colonies and may produce spores. As the aerial mycelium develops, Streptomyces colonies produce diverse secondary metabolites and synthesise antibiotics [1]. This differentiation cycle can be reproduced in laboratory conditions by growing

\footnotetext{
* Correspondence: philippe.mazodier@pasteur.fr

'Unité de Biologie des Bactéries Pathogènes à Gram-Positif, Institut Pasteur, CNRS URA 2172, 28 rue du Docteur Roux, 75724 Paris Cedex 15, France Full list of author information is available at the end of the article
}

Streptomyces cells on solid media. Most Streptomyces species do not form aerial mycelium or spores when in liquid media (e.g. S. coelicolor and S. lividans), and antibiotic production occurs in submerged cultures [2].

AdpA, also known as BldH, has been identified as a conserved major transcriptional regulator involved in the formation of aerial mycelia in various Streptomyces species [3-6]. AdpA is a member of the family of $\mathrm{AraC} /$ XylS regulator proteins that contain a C-terminal domain with two helix-turn-helix DNA-binding motifs; these features are strictly conserved in all Streptomyces AdpAs in the StrepDB database [7]. The N-terminal domain of AdpA is responsible for its dimerization and regulation $[8,9]$. Protein/DNA interaction experiments identified the following consensus AdpA-binding site in

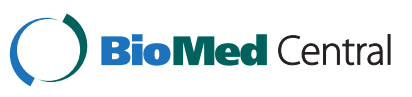

(c) 2014 Guyet et al.; licensee BioMed Central Ltd. This is an Open Access article distributed under the terms of the Creative Commons Attribution License (http://creativecommons.org/licenses/by/2.0), which permits unrestricted use, distribution, and reproduction in any medium, provided the original work is properly credited. The Creative Commons Public Domain Dedication waiver (http://creativecommons.org/publicdomain/zero/1.0/) applies to the data made available in this article, unless otherwise stated. 
S. griseus: 5' -TGGCSNGWWY-3' (with S: G or C; W: A or T; Y: T or C; N: any nucleotide) [10].

AdpA was discovered and has mostly been studied in S. griseus, in which it was first shown to activate expression of about thirty genes directly. They include genes encoding secreted proteins (e.g. proteases), a sigma factor (AdsA), a subtilisin inhibitor (SgiA), SsgA which is essential for spore septum formation and the AmfR transcriptional regulator involved in production of AmfS (known as SapB in S. coelicolor), a small hydrophobic peptide involved in the emergence of aerial hyphae [11,12]. AdpA also plays a role in secondary metabolism and directly activates streptomycin biosynthesis [3].

Proteomic, transcriptomic and ChIP-sequencing analyses revealed that, in fact, several hundred genes are under the control of S. griseus AdpA and that AdpA acts as transcriptional activator as well as repressor [12-15]. In $S$. coelicolor, few genes have been identified as being directly regulated by AdpA: sti1 (sgiA orthologs), ramR (amfR orthologs), clpP1 (encoding a peptidase) [16] and $w b l A$ (encoding a transcriptional regulator) [15].

The regulation of $a d p A$ gene expression is complex and various mechanisms have been described [17]. AdpA represses its own gene expression in S. griseus [18] whereas it activates its own transcription in S. coelicolor [16]. In several Streptomyces species, the binding of $\gamma$-butyrolactones to a $\gamma$-butyrolactone receptor represses the $a d p A$ promoter $[19,20]$. In S. coelicolor, BldD represses adpA expression [21]. At the translational level, a feedback-control loop regulates levels of AdpA and AbsB (a RNAse III) in S. coelicolor [22,23]. A positive feedback loop between AdpA and BldA, the only tRNA able to read the UUA codon present in all $\operatorname{adpA}$ mRNA, has been demonstrated in S. griseus [22,23]. In $S$. coelicolor, adpA expression is constant during growth in liquid media [4] whereas on solid media, $\operatorname{adp} A$ is strongly expressed before aerial hyphae formation and AdpA is most abundant during the early aerial mycelium stage $[4,16]$.

Even though AdpA plays a major role in development of Streptomyces spp., little is known about the pathways it controls in S. lividans, a species closely related to $S$. coelicolor and whose genome has recently been sequenced [24]. We have recently shown that in S. lividans AdpA directly controls sti1 and the clpP1clpP2 operon, encoding important factors for Streptomyces differentiation; we also found interplay between AdpA and ClpP1 [25]. Here, we report microarray experiments, quantitative real-time PCR (qRT-PCR), in silico analysis and protein/ DNA interaction studies that identify other genes directly regulated by AdpA in S. lividans. Finally, in silico genome analysis allowed the identification of over hundred genes that are probably directly activated or repressed by AdpA in S. lividans. These findings and observations reveal new AdpA-dependent pathways in S. lividans.

\section{Methods}

Bacterial strains, growth conditions and media

S. lividans 1326 was obtained from the John Innes Culture Collection. In this S. lividans background, we constructed an $\operatorname{adp} A$ mutant in which $\operatorname{adp} A$ was replaced with an apramycin-resistance cassette [25].

Streptomyces was grown on NE plates [26] and in YEME liquid medium [27] in baffled flasks. MS medium was used for sporulation experiments [27]. Apramycin was added to final concentrations of $25 \mu \mathrm{g} \mathrm{mL} \mathrm{m}^{-1}$ to solid media and $20 \mu \mathrm{g} \mathrm{mL}^{-1}$ to liquid media as appropriate.

\section{Microarray experiments}

$S$. lividans microarrays were not available, so $S$. coelicolor oligonucleotide arrays covering most open reading frames (ORFs) of the genome (for array coverage and design, see $[28,29])$ were used. Aliquots of $60 \mathrm{~mL}$ of liquid YEME medium were inoculated with about $10^{8}$ spores and incubated at $30^{\circ} \mathrm{C}$ with shaking at $200 \mathrm{rpm}$ until early stationary phase (about $30 \mathrm{~h}$ of growth). Samples of $12 \mathrm{~mL}$ of culture (at $\mathrm{OD}_{450 \mathrm{~nm}}=2.3$, corresponding to time point $\mathrm{T}$ on Figure 1a) were then collected and RNA extracted as previously described [30]. RNA quality was assessed with an Agilent 2100 Bioanalyser (Agilent Technologies). RNA indirect labelling and array hybridization were performed as described [31] and hybridized microarrays were scanned with a Genepix 4000A scanner (Molecular Devices).

\section{Statistical analysis of array data}

$\mathrm{R}$ software [32] was used for normalization and differential analysis. A Loess normalization [33] was performed on a slide-by-slide basis (BioConductor package marray; [34]). A paired $t$-test was used for differential analysis. Variance estimates for each gene were computed under the hypothesis of homoscedasticity, together with the Benjamini and Yekutieli $P$-value adjustment method [35]. Only genes with a significant $(P$-value $<0.05)$ fold change $(\mathrm{Fc})$ were taken into consideration. Empty and flagged spots were excluded, and only genes with no missing values were analysed. A few genes which displayed excessive variation were analysed using the Vmixt method from the VarMixt package [36]. We defined our cut-off for microarray data acquisition as Fc $<0.625$ or $\mathrm{Fc}>1.6$ with $P$-value $<0.05$. The genome of S. lividans 1326 was sequenced only recently [24], so we used the StrepDB database [7], and in some cases a basic local alignment search tool (Blast), to identify $S$. lividans orthologs (SLI gene number) of S. coelicolor genes. We also used the protein classification scheme for the $S$. coelicolor genome available on the Welcome Trust Sanger Institute database [37].

\section{qRT-PCR analysis}

Oligonucleotide pairs specific for cchA (SLI0459), cchB (SLI0458), SLI0755, SLI6586, ramR (SLI7029), hyaS 


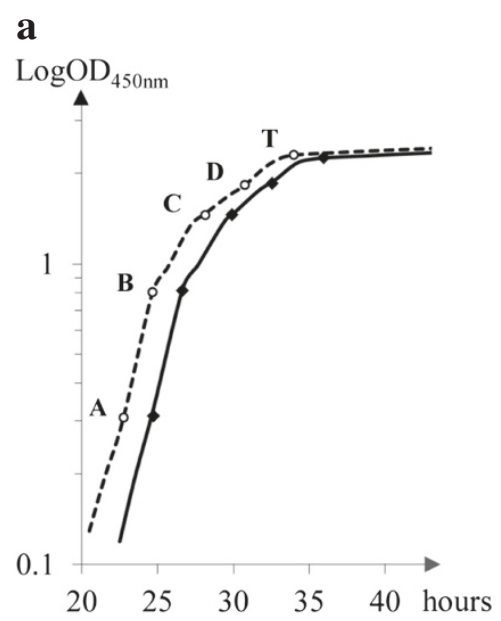

b

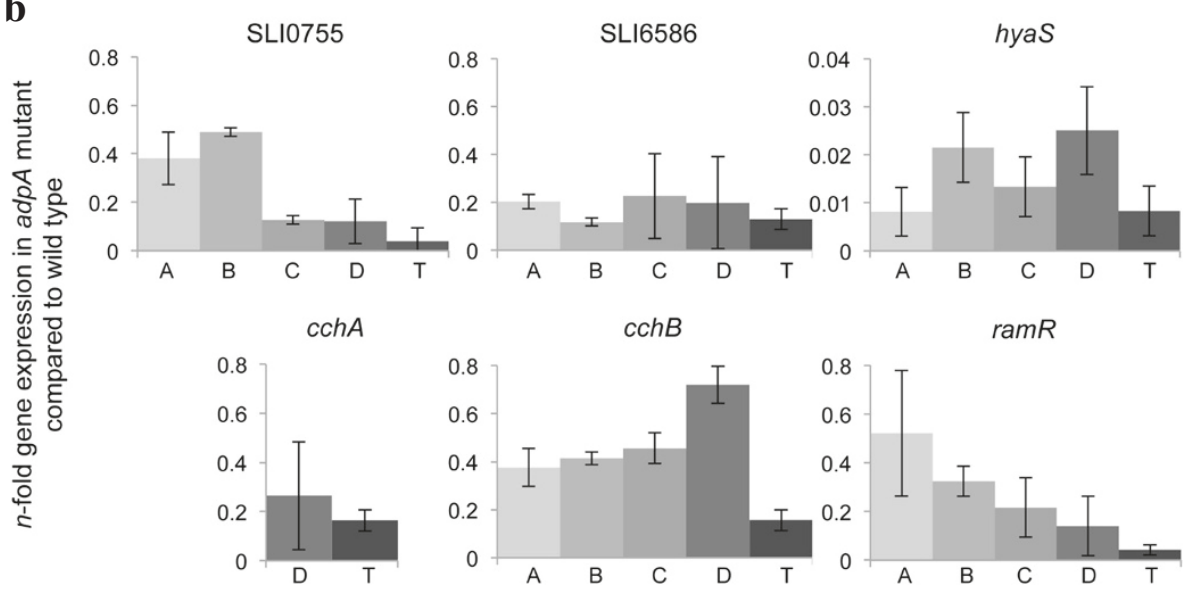

Figure 1 Effects of S. lividans adpA mutation on expression of selected genes. a. Growth curve of wild-type S. lividans (dashed line) and adpA mutant (solid line) in YEME liquid medium at $30^{\circ} \mathrm{C}$ with shaking at $200 \mathrm{rpm}$ as followed by measuring absorbance at $450 \mathrm{~nm}$. A, B, C, D and $T$ indicate the time points when cultures were harvested for RNA extraction. Microarray experiments were performed on RNA samples extracted at time T. b. Change in gene expression S. lividans adpA mutant compared to the wild-type at each time point of growth. RNA was extracted from S. lividans wild-type 1326 and adpA mutant cells cultivated in liquid YEME medium after various times of growth $\left(\mathrm{OD}_{450 \mathrm{~nm}}\right.$ of $0.3,0.8,1.5,1.9$ and 2.3, respectively, at time points A, B, C, D and T). Relative amounts of SLI0755, SLI6586, hyaS, cchA, cchB, ramR PCR product were measured by qRT-PCR. At each time point of growth, gene expression levels were normalized using hrdB as an internal reference and are indicated in this figure as the $n$-fold change in adpA mutant compared to the wild type. Results are expressed as means and standard deviations of at least three replicates. Data are representative of at least two independent experiments for each strain at each growth time. Note that a different scale is used for hyas.

(SLI7885) and $h r d B$ (SLI6088, MG16-17) (Additional file 1: Table S1) were designed using the BEACON Designer software (Premier BioSoft). RNA samples were extracted from cultures in YEME liquid medium at $\mathrm{OD}_{450 \mathrm{~nm}}$ values of about $0.3,0.8,1.5,1.9$ and 2.3 (time points A, B, C, D and $\mathrm{T}$, respectively). Aliquots of $20 \mu \mathrm{g}$ of RNA were treated twice with 2 Units of DNase I with the TURBO DNA-free reagent (Ambion) for $30 \mathrm{~min}$ at $37^{\circ} \mathrm{C}$. Reverse transcription and quantitative real-time PCR were performed as previously described [25]. PCRs involved a hybridization step of $55^{\circ} \mathrm{C}$, except for $\mathrm{ramR}$, SLI0755 and $\operatorname{cch} B$ where a temperature of $58^{\circ} \mathrm{C}$ was used. Each assay was performed in triplicate and repeated with at least two independent RNA samples. The critical threshold cycle $\left(C_{T}\right)$ was defined for each sample. The relative amounts of cDNA for the tested genes were normalized to that of the $h r d B$ gene transcript which did not vary under our experimental conditions (and thus served as an internal standard). The change ( $n$-fold) in a transcript level was calculated using the following equations: $\Delta C_{T}=C_{T \text { (test DNA) }}-C_{T \text { (reference cDNA), }}$, $\Delta \Delta C_{T}=\Delta C_{T(\text { target gene) }}-\Delta C_{T(\mathrm{hrdB})}$, and ratio $=2^{-\Delta \Delta C_{T}}$ [38]. Student's $t$ test was used to evaluate the significance of 
differences between the expression level of tested genes and that of a reference gene. A $P$-value $<0.05$ was considered significant.

\section{In silico analysis and electrophoretic mobility shift assays (EMSA)}

Several AdpA-binding site sequences, identified in S. griseus by DNase I footprinting experiments [10,13,18,23], were used with the PREDetector software (version 1.2.3.0) [39] to generate a $S$. griseus matrix [25]. This matrix was used with the $S$. coelicolor genome sequence (the $S$. lividans genome sequence was not available during the course of this study and is still not available on PREDetector software) to identify putative AdpA-binding sites upstream from S. lividans AdpA-dependent genes (scores > 3). The StrepDB database [7] and Blast were used to identify $S$. lividans, S. coelicolor and S. griseus ortholog gene names.

Radioactively labelled DNA fragments (180 bp to $496 \mathrm{bp}$ ) corresponding to promoter regions of putative S. lividans AdpA-regulated genes were obtained by PCR. Primers (named GSgene in Additional file 1: Table S1) were used to amplify the promoter regions of $\operatorname{cch} A$ (opposite orientation to $\operatorname{cch} B$ ), SLI0755, SLI6586 (opposite orientation to SLI 6587), ramR and hyaS as described elsewhere [25]. Purified radiolabelled fragments $(10,000 \mathrm{cpm})$ were then used with purified AdpA histidine-tagged protein (AdpA-His 6 ) in EMSA as previously described [25,40].

\section{Results}

Deletion of adpA affects the expression of hundreds of genes during early stationary phase

We had previously inactivated $a d p A$ in S. lividans and found that this $\operatorname{adp} A$ mutant failed to produce aerial mycelium on rich media and that its growth was comparable to that of the parental strain 1326 in liquid YEME medium at $30^{\circ} \mathrm{C}$ [25]. Expression studies with this $S$. lividans $\operatorname{adp} A$ mutant cultivated in liquid medium identified two differentiation-regulating factors (STI1 and the ClpP1ClpP2 peptidases) whose ORFs were under the direct control of AdpA [25]. We used transcriptome analysis of this $\operatorname{adpA}$ mutant to identify other AdpAdependent pathways in S. lividans; however, this analysis was performed using S. coelicolor microarrays [29] because the $S$. lividans genome sequence was not yet available [24] and the two species are very closely related [41]. Total RNA was isolated from S. lividans 1326 and $a d p A$ cells during early stationary phase (time point $\mathrm{T}$ in Figure 1a) because at this growth phase, $S$. coelicolor $a d p A$ is expressed [4]; also the expression of genes involved in secondary metabolism in a $S$. coelicolor bldA mutant [42], a strain defective for AdpA translation, starts to diverge from that in the wild-type.

Global gene expression in the mutant was compared to that in the parental strain. The expression of more than 300 genes was affected in the $a d p A$ mutant at early stationary phase (Table 1 and Additional file 2: Table S2): 193 genes were significantly down-regulated (1.6-to 30 -fold i.e. $0.033<\mathrm{Fc}<0.625$ ), and 138 were up-regulated (1.6-to 3.6-fold) with a $P$-value $<0.05$ (see Additional file 2: Table $\mathrm{S} 2$ for the complete data set). Theses genes encode proteins of several different classes according to the Welcome Trust Sanger Institute S. coelicolor genome database [37]: 72 of the genes are involved in metabolism of small molecules, including seven playing a role in electron transport (e.g. SLI0755-SLI0754, cydAB operons) (Table 1); 18 encode proteins involved in secondary metabolism, for example the cchA-cchF gene cluster (SLI04590454) involved in coelichelin biosynthesis [43] and the SLI0339-0359 cluster encoding the putative deoxysugar synthase/glycosyltransferase. Deletion of $a d p A$ in S. lividans also affected the expression of 32 genes involved in regulation including $\mathrm{ramR}$ (SLI7029), wblA (SLI3822), bldN (SLI3667), hrdD (SLI3556) and cutRS (SLI6134-35) $[1,6]$. Sixty-two genes involved in the cell envelope [37] were differentially expressed in the $\operatorname{adpA}$ mutant; they include hyaS (SLI7885) [44], chpE, chpH [1], SLI6586 and SLI6587 which were strongly down-regulated in the $a d p A$ mutant (Table 1). Thirty-nine genes encoding proteins involved in various cellular processes (osmotic adaptation, transport/binding proteins, chaperones, and detoxification) [37] were also deregulated in the absence of AdpA (Additional file 2: Table S2). The expression of 111 genes coding for proteins with unidentified or unclassified function was altered in the $a d p A$ mutant. Thus, deletion of $a d p A$ influenced the expression of a large number of genes involved in a broad range of metabolic pathways, and indeed other functions, in S. lividans.

\section{Identification of new AdpA-controlled genes}

To confirm that S. lividans AdpA controls the expression of genes identified as differentially expressed in microarray experiments, six genes were studied in more detail by qRTPCR. The six genes were selected as having biological functions related to Streptomyces development or the cell envelope (ramR [1], hyaS [44] and SLI6586 [37]) or primary or secondary metabolism (SLI0755, cchA, and $\operatorname{cch} B$ [43]), and for having very large fold-change values (Table 1). The genes in S. coelicolor and griseus orthologous to SLI6586 and SLI6587 encode secreted proteins $[12,42]$. The expression levels of these genes in S. lividans wild-type and $a d p A$ strains were measured after various times of growth in liquid YEME media (Figure 1b), as shown in Figure 1a.

The S. lividans hyaS gene was strongly down-regulated in the $\operatorname{adp} A$ mutant compared to the wild-type ( $\mathrm{Fc}<0.03$ ) (Figure 1b) as previously observed for the SCO0762 homolog also known as sti1 [25]. This suggests that hyaS expression is strongly dependent on S. lividans AdpA or an 
Table 1 Genes differentially expressed in S. lividans adpA mutant at early stationary phase in YEME medium ${ }^{\text {a }}$

\begin{tabular}{|c|c|c|c|c|c|}
\hline S. coelicolor gene ${ }^{\text {b }}$ & S. lividans gene ${ }^{c}$ & Other gene names $^{d}$ & Annotated function $^{b}$ & $\mathrm{Fc}^{\mathrm{e}}$ & Class or metabolism ${ }^{f}$ \\
\hline SCO0382 & SLI0340 & & $\begin{array}{l}\text { UDP-glucose/GDP-mannose } \\
\text { family dehydrogenase }\end{array}$ & 0.491 & Secondary (s. m.) \\
\hline SCO0383 & SLI0341 & & Hypothetical protein SCF62.09 & 0.527 & Secondary (s. m.) \\
\hline SCO0384 & SLI0342 & & Putative membrane protein & 0.611 & Secondary (s. m.) \\
\hline SCO0391 & SLI0349 & & Putative transferase & 0.613 & Secondary (s. m.) \\
\hline SCO0392 & SLI0350 & & Putative methyltransferase & 0.606 & Secondary (s. m.) \\
\hline SCO0394 & SLI0352 & & Hypothetical protein SCF62.20 & 0.518 & Secondary (s. m.) \\
\hline SCO0396 & SLI0354 & & Hypothetical protein SCF62.22 & 0.454 & Secondary (s. m.) \\
\hline SCO0397 & SLI0355 & & Putative integral membrane protein & 0.312 & Secondary (s. m.) \\
\hline SCO0399 & SLI0357 & & Putative membrane protein & 0.532 & Secondary (s. m.) \\
\hline SCO0494 & SLI0454 & $c c h F$ & $\begin{array}{l}\text { Putative iron-siderophore } \\
\text { binding lipoprotein }\end{array}$ & 0.615 & Secondary (s. m.) \\
\hline SCO0496 & SLI0456 & $\operatorname{cchD}$ & $\begin{array}{l}\text { Putative iron-siderophore permease } \\
\text { transmembrane protein }\end{array}$ & 0.505 & Secondary (s. m.) \\
\hline SCO0497 & SLI0457 & $\operatorname{cch} C$ & $\begin{array}{l}\text { Putative iron-siderophore permease } \\
\text { transmembrane protein }\end{array}$ & 0.492 & Secondary (s. m.) \\
\hline SCO0498 & SLI0458* & $\operatorname{cch} B$ & Putative peptide monooxygenase & 0.336 & Secondary (s. m.) \\
\hline SCO0499 & SLI0459* & $\operatorname{cch} A$ & Putative formyltransferase & 0.374 & Secondary (s. m.) \\
\hline SCO0762 & SLI0743 & stil, sgiA & Protease inhibitor precursor & 0.124 & (m. m.) \\
\hline SCO0773 & SLI0754 & soyB2 & Putative ferredoxin, Fdx4 & 0.098 & Electron transport (s. m.) \\
\hline SCO0774 & SLI0755* & & Putative cytochrome P450, CYP105D5 & 0.075 & Electron transport (s. m.) \\
\hline SCO0775 & SLI0756* & & Conserved hypothetical protein & 0.424 & Unknown function \\
\hline SCO1630-28 & SLI1934-32 & $\operatorname{rar} A B C, c \vee n A B C 9$ & Putative integral membrane protein & \pm 0.43 & Cell envelope \\
\hline SCO1674 & SLI1979 & $\operatorname{chpC}$ & Putative secreted protein & 0.564 & Cell envelope \\
\hline SCO1675 & SLI1980 & chpH & Putative small membrane protein & 0.237 & Cell envelope \\
\hline SCO1800 & SLI2108 & $c h p E$ & Putative small secreted protein & 0.256 & Cell envelope \\
\hline SCO2780 & SLI3127 & desE & Putative secreted protein & 1.757 & Cell envelope \\
\hline SCO2792 & SLI3139 & bldH, adpA & araC-family transcriptional regulator & 0.383 & Regulation \\
\hline SCO2793 & SLI3140 & ornA & Oligoribonuclease & 1.966 & (m. m.) \\
\hline SCO3202 & SLI3556 & $\operatorname{hrdD}$ & RNA polymerase principal sigma factor & 2.499 & Regulation \\
\hline SCO3323 & SLI3667 & $b / d N$, ads $A$ & Putative RNA polymerase sigma factor & 0.389 & Regulation \\
\hline SCO3579 & SLI3822 & WblA & Putative regulatory protein & 0.310 & Regulation \\
\hline SCO3945 & SLI4193 & cydA & Putative cytochrome oxidase subunit I & 3.386 & Electron transport (s. m.) \\
\hline SCO3946 & SLI4194 & $c y d B$ & Putative cytochrome oxidase subunit II & 3.594 & Electron transport (s. m.) \\
\hline SCO4114 & SLI4345 & & Sporulation associated protein & 0.487 & Cell envelope \\
\hline SCO5240 & SLI5531 & wblE & Hypothetical protein & 2.246 & Unknown function \\
\hline SCO5862-63 & SLI6134-35 & cutRS & Two-component regulator/sensor & \pm 1.82 & Regulation \\
\hline SCO6197 & SLI6586* & & Putative secreted protein & 0.147 & Cell envelope \\
\hline SCO6198 & SLI6587* & & Putative secreted protein & 0.618 & Cell envelope \\
\hline SCO6685 & SLI7029* & $\operatorname{ramR}, a m f R$ & $\begin{array}{l}\text { Putative two-component system } \\
\text { response regulator }\end{array}$ & 0.624 & Regulation \\
\hline
\end{tabular}


Table 1 Genes differentially expressed in S. lividans adpA mutant at early stationary phase in YEME medium ${ }^{\mathrm{a}}$ (Continued)

\begin{tabular}{lllll}
\hline SCO7400-398 & SLI7619-17 & cdtCBA & Putative ABC-transport protein & Cell process \\
SC07657 & SLI7885* & hyaS & Putative secreted protein & Cell envelope \\
SC07658 & detected & & Hypothetical protein SC10F4.31 & 0.1033 \\
\hline
\end{tabular}

${ }^{a}$ Gene expression in the $S$. lividans adpA mutant was compared to that in the wild-type, using S. coelicolor microarrays. Table 1 shows a selected subset of the genes (see Additional file 2: Table S2 for the complete list). The genes presented here were further studied or are discussed in the text because of their role in Streptomyces primary or secondary metabolism [1,6,17].

${ }^{\mathrm{b}}$ Gene names for S. coelicolor (SCO) and S. lividans (SLI) and annotated function are from the StrepDB database [7].

${ }^{\mathrm{C}} \mathrm{S}$. coelicolor microarrays were used for transcriptome analysis of the S. lividans adpA mutant (the complete microarray data set is presented in Additional file 2: Table S2). The S. lividans genome sequence was recently made available [24] and SLI ortholog gene numbers were identified as SCO gene orthologs with StrepDB database [7]. The expression of genes shown in bold was analysed by qRT-PCR. Intergenic DNA regions between genes labelled with asterisks were analyzed by EMSA (Figure 2). A SCO7658-orthologous sequence (98\% nucleotide identity according to BLAST) was detected in S. lividans, downstream from hyaS, but it was not annotated as a S. lividans coding DNA sequence (CDS). However our microarray data suggest that this sequence is indeed a CDS or alternatively that the S. lividans hyaS CDS is longer than annotated.

"SCO genes and their S. griseus orthologs studied and described under another name found on StrepDB database [7] or see "References".

e Fold change (Fc) in gene expression in the S. lividans adpA mutant with respect to the parental strain with $P$-value $<0.05$, as calculated by Student's $t$-test applying the Benjamini and Hochberg multiple testing correction. \pm indicates average Fc of some gene operons (see Additional file 2: Table S2 for details). ${ }^{f}$ From a protein classification scheme for the S. coelicolor genome available from the Welcome Trust Sanger Institute database [37]: macromolecule metabolism (m. m.), small molecule metabolism (s. m.).

AdpA-dependent regulator. SLI0755, SLI6586 and ramR, were also expressed at a lower level in the $\operatorname{adpA}$ mutant than wild-type, particularly after mid-exponential phase (Figure $1 \mathrm{~b}$, times $\mathrm{C}, \mathrm{D}$ and $\mathrm{T}$ ); $\operatorname{cch} B$ seemed to be mostly affected by AdpA during stationary phase (Figure 1b, time $\mathrm{T})$. The expression of $c$ chA was strongly down-regulated by the absence of AdpA at times D and T (Figure 1b): note that despite repeated efforts, $\operatorname{cch} A$ expression could not be detected in samples corresponding to times $\mathrm{A}$ to $\mathrm{C}$ for unknown reasons. The findings for gene expression as determined by microarrays and by qRT-PCR were consistent, with the exception of those for $r a m R$. The expression of ramR observed by qRT-PCR at time $\mathrm{T}$ differed from that determined in microarray experiments (Table 1), suggesting that some of our microarray data are flattened. Nevertheless, these qRT-PCR experiments confirmed that the expression of the six selected genes is indeed AdpAdependent in S. lividans at every growth time studied.

\section{Direct binding of AdpA to the promoter regions of S. lividans AdpA regulon members}

To determine whether S. lividans AdpA directly controls these genes, we searched for potential AdpA-binding sites in their promoter regions in silico. A consensus AdpAbinding sequence $\left({ }^{5}\right.$ TGGCSNGWWY $\left.{ }^{3}\right)$ has been established in S. griseus, and AdpA can bind up to five sites between positions $-260 \mathrm{bp}$ and $+60 \mathrm{bp}$ with respect to the transcriptional start point of the target gene [10]. BLAST analysis revealed that the S. griseus AdpA DNAbinding domain is conserved in S. coelicolor and S. lividans AdpAs (data not shown) suggesting that all three species share the same AdpA-binding consensus sequence.

The DNA sequences upstream from the $S$. coelicolor ramR and hyaS genes and the intergenic region between the divergently transcribed genes $\operatorname{cch} A / \operatorname{cch} B$, SCO0774/ SCO0775 and SCO6197/SCO6198 were analyzed using
PREDetector software [39] and a matrix was generated with identified S. griseus AdpA-binding sequences [10,23,25]. Between three and nine putative AdpA-binding sites were detected within the promoter region of the S. coelicolor genes and by analogy in orthologous S. lividans AdpAdependent genes (Table 2, location with respect to translation start point). During the course of this study, the $S$. lividans 1326 genome sequence became available [24] (but not in a form suitable for analysis with PREDetector (version 1.2.3.0) [39]) and its analysis suggested that the position and composition of AdpA-binding sites were different from those predicted. The putative AdpA-binding sites of S. lividans $\operatorname{cch} A / \operatorname{cch} B$ at $-101 \mathrm{nt}$ and $-86 \mathrm{nt}$ are GGGCCGGTTC and TGGCTGGAAC, respectively. The AdpA-binding sites located upstream of SLI0755, SLI6586, and hyaS differ from their S. coelicolor orthologs (see Table 2, changes in the location from translation start site are indicated in bracket).

We used EMSA to test whether S. lividans AdpA binds to predicted S. lividans AdpA-binding sequence. Recombinant purified AdpA-His ${ }_{6}$ bound to the promoter region of S. lividans sti1 (SCO0762 homolog), an AdpAdependent gene, whereas an excess of AdpA- $\mathrm{His}_{6}$ (up to 34 pmoles) did not bind to the promoter of SLI4380 (SCO4141 homolog), a gene that is not controlled by $S$. lividans AdpA. This suggests that the binding of AdpA with the promoter of genes tested in our previous study was specific [25]. AdpA-His ${ }_{6}$ was able to bind to the promoter regions of all S. lividans AdpA-dependent genes tested (Table 2, Figure 2), although with different affinities. For SLI6586/SLI6587, ramR and hyaS, displacement of the DNA fragment to the slower migrating protein-DNA complex was nearly complete with amounts of AdpA of less than 11 pmoles (Figure 2, lane 2). For $\operatorname{cch} A / \operatorname{cch} B$ and SLI0755/SLI0756, larger amounts of AdpA were necessary for near complete displacement of the DNA probe to a 
Table 2 AdpA-binding sites identified in silico in the promoter regions of S. lividans AdpA-dependent genes ${ }^{\mathrm{a}}$

\begin{tabular}{|c|c|c|c|c|c|c|}
\hline $\begin{array}{l}\text { S. coelicolor gene } \\
\text { (S. lividans gene) }^{\mathbf{b}}\end{array}$ & Putative AdpA-binding site $^{c}$ & \multicolumn{2}{|c|}{$\begin{array}{l}\text { Position (bp) with respect } \\
\text { to translation start site }\end{array}$} & \multirow[t]{2}{*}{ Strand location $^{d}$} & Scores ${ }^{\mathrm{e}}$ & Sites in EMSA probes ${ }^{\dagger}$ \\
\hline \multirow[t]{4}{*}{ cchA/cchB* } & TGGCCGGATT" & $-425^{\#}$ & & & 9.30 & + \\
\hline & TGGCGACATT" & $-254^{\#}$ & & CS & 5.19 & + \\
\hline & GGGCCGATTC $\left(G^{7 \text { th }}\right)$ & -101 & & $\mathrm{CS}$ & 4.99 & + \\
\hline & TGGCTCGAAT (C $\left.{ }^{10 \text { th }}\right)$ & -86 & & NCS & 6.91 & + \\
\hline \multirow[t]{9}{*}{$\operatorname{ramR}$} & GTGCCGGTTC & -464 & & NCS & 3.37 & - \\
\hline & TGGCGCGAAA & -384 & & NCS & 6.42 & + \\
\hline & CGGCCGAAAA & -358 & & NCS & 5.85 & + \\
\hline & GGGCGGGTTC & -280 & & NCS & 5.08 & + \\
\hline & TGGCCAGGAC & -279 & & $\mathrm{CS}$ & 3.86 & + \\
\hline & GGGCGGATAA & -184 & & NCS & 3.87 & + \\
\hline & TGTCGTGTTC & -95 & & CS & 4.83 & - \\
\hline & CGGCGGAACA & -81 & & NCS & 3.15 & - \\
\hline & TGGCCCGAAC & -30 & & CS & 7.23 & - \\
\hline SCO0774/SCO0775* & CGGCGCGTTC & -268 & $(-226)$ & CS & 4.25 & - \\
\hline \multirow[t]{6}{*}{ (i.e. SLI0755/SLI0756) } & GGACGGGAAC & -253 & $(-211)$ & NCS & 3.37 & + \\
\hline & GGGCGCGATC & -207 & $(-165)$ & $\mathrm{CS}$ & 4.53 & + \\
\hline & TGGCGCGATC & -170 & $(-128)$ & NCS & 6.90 & + \\
\hline & CGGCCAGTCT & -110 & $(-68)$ & CS & 3.06 & + \\
\hline & TGGCCGAACT & -84 & $(-42)$ & CS & 6.20 & - \\
\hline & CGGCCAGATC & -79 & $(-37)$ & NCS & 5.84 & - \\
\hline SCO6197/SCO6198* & GGTCCGGACA & -499 & $\left(-547^{\sim}\right)$ & CS & 4.98 & - \\
\hline \multirow[t]{5}{*}{ (i.e. SLI6586/SLI6587) } & TGACCAGAAG & -414 & $\left(-462^{\sim}\right)$ & CS & 3.82 & + \\
\hline & TGGCCGAGTT & -362 & $\left(-410^{\sim}\right)$ & CS & 5.06 & + \\
\hline & GTTCCTGCAA & -297 & $\left(-345^{\sim}\right)$ & NCS & 3.50 & + \\
\hline & GGGCTGAAAC & -271 & $\left(-319^{\sim}\right)$ & NCS & 4.77 & + \\
\hline & TGGCTGAATT & -116 & $(-164)$ & CS & 7.85 & + \\
\hline \multirow[t]{3}{*}{ hyas } & TGGCCGGATC & -130 & $(-129)$ & NCS & 8.90 & + \\
\hline & CGGCCATTTC & -124 & $(-123)$ & $\mathrm{CS}$ & 3.05 & + \\
\hline & TGTCCAGAAG & -101 & $(-100)$ & NCS & 4.48 & + \\
\hline
\end{tabular}

${ }^{\mathrm{a}}$ In silico analysis of the $S$. coelicolor genome using PREDetector software (version 1.2.3.0, the $S$. lividans database was not available at the time this analysis was performed) [39] to analyse orthologs of S. lividans AdpA-dependent genes. The S. coelicolor AdpA-binding sites identified were checked for their conservation and location using the S. lividans genome StrepDB database [7] (see legend c).

${ }^{\mathrm{b}}$ Genes are named according to the StrepDB database [7]. *binding sites located between S. coelicolor genes transcribed in the opposite orientation.

'Putative S. coelicolor AdpA-binding sites were found in silico with PREDetector [39]; "putative site located in the upstream from the CDS of cchB. The site location given corresponds to the position of first nucleotide most distant from the translation start point of the first gene named. The positions of some sites are not the same for the S. lividans orthologs as indicated in brackets (S. lividans StrepDB database [7]). putative sites are in the CDS of SLI6587. Predicted CDS diverge between SLI6586 and SLI6587 locus and their orthologs SCO6197 and SCO6198, resulting in a smaller intergenic region in S. lividans.

${ }^{d} \mathrm{CS}$, coding strand; NCS, non coding strand with reference to the first gene named in the $S$. coelicolor gene column.

${ }^{\text {e}}$ Scores given by PREDetector software for S. coelicolor genes [39].

${ }^{\mathrm{f}} \mathrm{Sites}$ present $(+)$ or absent $(-)$ in the S. lividans DNA probes used in EMSA experiments.

protein-DNA complex. In a competition EMSA performed on SLI6586/6587 with an excess of the corresponding unlabelled probe, AdpA-binding to the labelled probe decreased (data not shown). We also tested a hyaS promoter in which one (highest score) of the three putative AdpAbinding sites was mutated (at position -134 to -129 , see Additional file 3: Figure S1a): the affinity of AdpA for this promoter region was reduced and one protein-DNA complex disappeared (Additional file 3: Figure S1b). These results suggest that one dimer of AdpA binds the adjacent sites -129 and -123 of S. lividans hyaS promoter and another dimer binds the -100 site resulting in the formation of the two DNA-AdpA complexes depicted in Figure 2.

These EMSA experiments demonstrated that S. lividans AdpA directly binds to five intergenic regions and confirmed the in silico prediction presented in Table 2. S. 


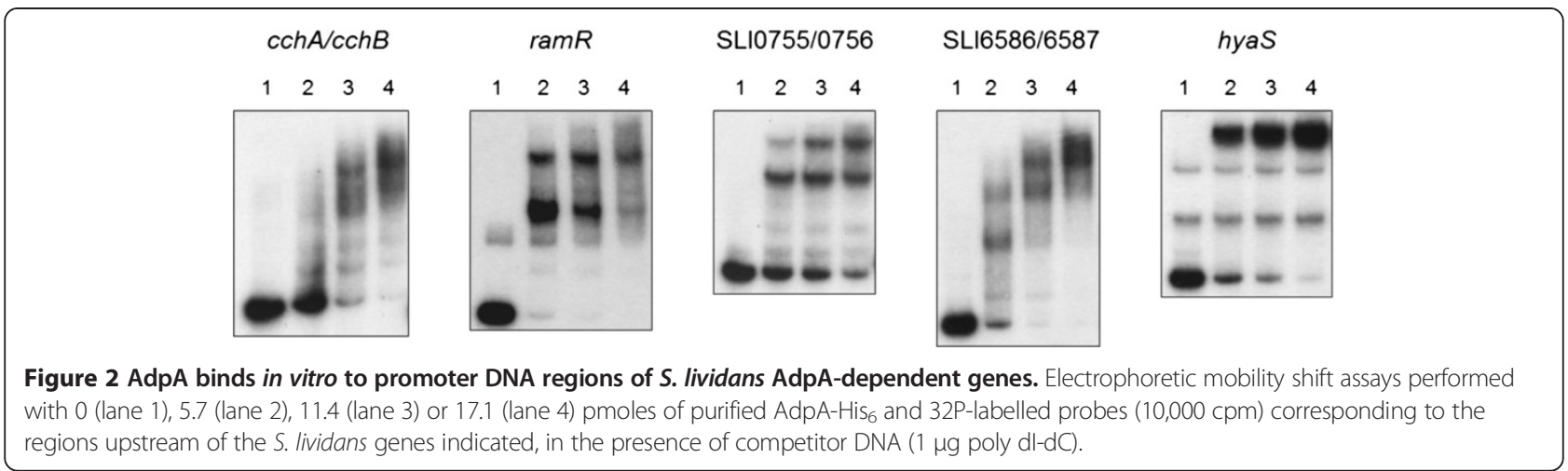

lividans AdpA directly regulates at least the six AdpAdependent genes listed above and identified by microarrays and qRT-PCR analysis. These newly identified targets highlight the pleiotropic role of S. lividans AdpA: it is involved in primary (SLI0755) and secondary (cchA, cchB and hyaS) metabolisms, in regulation (ramR), and in cell development (hyaS, ramR and SLI6586).

\section{Discussion}

AdpA, a transcriptional regulator of the AraC/XylS family, is involved in the development and differentiation of various Streptomyces [3-5,25]. We report here the first identification of several pathways directly regulated by AdpA in S. lividans cultivated in liquid rich medium.

Inactivation of $a d p A$ in S. lividans affected the expression of approximately 300 genes. This large number was expected in the light of the size of the S. griseus AdpA regulon [14]. Although $a d p A$ mutant growth was comparable to that of the parental strain in YEME liquid medium, the expression of around 200 genes involved in primary metabolism was influenced by adpA deletion. These genes encode proteins involved in the major biosynthesis pathways for amino acids (class 3.1. in Additional file 2: Table S2) [37], and in energy metabolism (class 3.5.) including glycolysis, pentose phosphate, pyruvate dehydrogenase pathways, as well as in electron transport (e.g. CydAB cytochrome oxidase, CYP105D5 and Fdx4 involved in fatty acid hydroxylation and encoded by SLI0755-0754 [45]). Other S. lividans AdpA-regulated genes influence Streptomyces development on solid media (e.g. those for RamR, chaplins Chp, BldN, WblA, WblE, HyaS and ClpP1ClpP2 peptidases) (Table 1) $[1,6,16,25,44]$. S. lividans AdpA also influences the expression of 18 genes involved in secondary metabolism such as coelichelin biosynthesis (cch genes in Table 1) [43] and also genes described to affect metabolic differentiation (HyaS, CutRS, WblA, DesE, and CdtCBA) (Table 1) $[15,17,42,44]$. Consistently with transcriptomic studies in S. griseus, these observations suggest that AdpA is a pleiotropic transcriptional regulator in S. lividans.
We demonstrate that $S$. lividans AdpA directly activates $c c h B$, SLI0755 and hyaS. As a result of their cotranscription with these genes, the expression of $c c h C D$, SLI0754 and SCO7658-ortholog genes is AdpA-dependent in S. lividans (Table 1). SLI0756 is probably a directly AdpA-regulated gene because its promoter DNA region is shared with SLI0755-SLI0754 operon, which is transcribed in the opposite direction and directly regulated by AdpA (Table 1, Figure 2).

AdpA directly regulates the genes ramR and sti1 in $S$. lividans (this study) [25] and in the closely related species S. coelicolor [16]. In an S. coelicolor adpA mutant, levels of sti1 and ramR expression were lower than in the wild-type strain following growth for $48 \mathrm{~h}$ in a minimal agar medium [16]. In vitro experiments showed a high affinity of AdpA with a S. coelicolor sti1 probe [16], consistent with our results with S. lividans sti1 [25]. However, AdpA had a lower affinity to $S$. coelicolor ramR (with promoter region $-302 \mathrm{nt}$ to $+73 \mathrm{nt}$ with respect to the translation start site) than S. lividans ramR (Figure 2, with the promoter region $-440 \mathrm{nt}$ to $-181 \mathrm{nt})$. When we used a S. lividans ramR probe carrying the promoter region from $-201 \mathrm{nt}$ to $+66 \mathrm{nt}$, we observed that less than half the probe was shifted (data not shown). Therefore, the predicted sites for $\mathrm{ramR}$ promoter at positions -384 and -358 (Table 2) may have the greatest affinity for AdpA (Figure 2). Of the genes analysed by qRT-PCR, the $\operatorname{ramR}$ gene was that for which the observed expression was the least consistent with the microarray findings, even through the same sample was used for these analyses. This suggests that the expression of genes close to the cut-off we applied to the microarray data will need further investigation by qRT-PCR.

Among the 28 genes identified as direct targets of AdpA in S. griseus, 13 have no orthologous gene in $S$. lividans and the orthologous genes of six are not under the control of $S$. lividans AdpA in our conditions. In addition to ramR (amfR) and stil (sgiA), hyaS (SGR3840) is also a directly AdpA-regulated gene that is conserved in the S. lividans and S. griseus AdpA regulons [12,25]. In $S$. 
Table 3 Genes putatively directly regulated by S. lividans AdpA in liquid rich medium ${ }^{\text {a }}$

\begin{tabular}{|c|c|c|c|c|c|c|c|c|}
\hline Gene $^{b}$ & Gene $^{\text {b }}$ & Gene $^{\mathbf{b}}$ & Gene name $^{\text {b }}$ & cis-element $^{c}$ & Score $^{c}$ & Position ${ }^{c}$ & $\mathrm{Fc}^{\mathrm{d}}$ & Class $^{\mathrm{e}}$ \\
\hline \multicolumn{9}{|c|}{ Probably directly activated by S. lividans AdpA: } \\
\hline SCO $2921^{*}$ & Detected & SGR4618 & adbS3-orfa & tttgcggaca & 4.62 & -260 & 0.196 & c. e. \\
\hline SCO0494 & SLI0454 & SGR6714 & cchF & tgtcgcgeca & 4.36 & -28 & 0.615 & s. m. \\
\hline SCO0929 & SLI1160 & $S G R 710$ & & tggecggacg & 5.19 & -201 & 0.419 & u. f. \\
\hline SCO1565 & SLI1668 & SGR5973 & glpQ1 & cggccggaac & 6.75 & -82 & 0.531 & c. e. \\
\hline SCO1630 & SLI1934 & SGR1063 & cvn9, rarA & tgtcgggatc & 6.71 & -74 & 0.505 & c. e. \\
\hline SCO1674 & SLI1979 & SGR5829 & $\operatorname{chpC}$ & cggcggaatc & 5.69 & -154 & 0.564 & c. e. \\
\hline SCO1800 & SLI2108 & SGR5696 & chpE & cggccggacc & 4.69 & -65 & 0.256 & c. e. \\
\hline SCO1968 & SLI2284 & SGR5556 & glpQ2 & cattcagcct & 3.75 & -92 & 0.537 & m. m. \\
\hline SCO2792 & SLI3139 & SGR4742 & adpA bldH & gaaccggeca & 8.09 & -148 & 0.383 & r. \\
\hline SCO3323 & SLI3667 & SGR4151 & bldN, adsA & gttccggtca & 6.38 & -469 & 0.389 & r. \\
\hline SCO3579* & SLI3822 & SGR3340 & wbla & tggeccgaac & 7.23 & -135 & 0.31 & $r$. \\
\hline SCO3917* & SLI4175 & SGR3663 & & ctttcggcca & 6.52 & -72 & 0.504 & u. f. \\
\hline SCO4113 & SLI4344 & SGR3901 & & aaacccgtca & 5.64 & -52 & 0.568 & u. f. \\
\hline SCO4114* & SLI4345 & SGR3902 & & tggcgggatt & 8.66 & -117 & 0.487 & c. p. \\
\hline SCO4164 & SLI4405 & SGR3965 & cysA & gttgccgcca & 5.70 & -170 & 0.483 & s. m. \\
\hline SCO4295* & SLI4532 & SGR3226 & $\mathrm{scoF}_{4}$ & attctcgeca & 7.13 & -193 & 0.217 & c. p. \\
\hline SCO4761 & SLI5031 & SGR2770 & groEs & aaccccgccg & 3.31 & -197 & 0.401 & c. p. \\
\hline SCO4762 & SLI5032 & SGR2769 & groEL1 & ttgccgtata & 4.40 & -44 & 0.44 & c. p. \\
\hline SCO4768 & SLI5039 & SGR2759 & bldM & aatctagccg & 5.52 & -292 & 0.586 & r. \\
\hline SCO5101 & SLI5379 & SGR2456 & & cggcgggaac & 6.11 & -28 & 0.584 & u. f. \\
\hline SCO6004 & SLI6392 & SGR1503 & & cggccgcatt & 5.21 & -292 & 0.603 & c. e. \\
\hline SCO6096* & SLI6490 & SGR1397 & & catcgcgeca & 5.56 & -147 & 0.557 & c. e. \\
\hline SCO7550 & SLI7772 & - & glpQ3 & gaaccggtca & 5.88 & -117 & 0.334 & c. e. \\
\hline \multicolumn{9}{|c|}{ Probably directly repressed by S. Iividans AdpA: } \\
\hline SCO1684 & SLI1989 & SGR5819 & & gaatgcgcca & 5.36 & -161 & 1.626 & u. f. \\
\hline SCO1776* & SLI2080 & SGR5721 & pyrG & cttccggeca & 7.25 & -170 & 1.744 & s. m. \\
\hline SCO1821 & SLI2130 & SGR5674 & moaA & cggcccgaac & 5.39 & -61 & 1.679 & s. m. \\
\hline SCO1864 & SLI2175 & SGR5635 & ectA & atttcggaca & 6.71 & -203 & 2.903 & c. p. \\
\hline SCO1865 & SLI2176 & SGR5634 & ectB & cggecgggac & 3.24 & -78 & 3.154 & c. p. \\
\hline SCO1867 & SLI2178 & SGR5632 & ectD & gaagtggcca & 4.62 & -3 & 3.029 & n. c. \\
\hline SCO3123 & SLI3480 & SGR4383 & prsA2 & tgaccggaaa & 6.21 & \# & 1.891 & s. m. \\
\hline SCO3202 & SLI3556 & SGR4276 & $\operatorname{hrdD}$ & aatccggaca & 7.75 & -145 & 2.499 & r. \\
\hline SCO3811 & SLI4062 & SGR3768 & dacA & tatccggacg & 5.34 & -175 & 1.628 & c. e. \\
\hline SCO3945 & SLI4193 & SGR3646 & $c y d A$ & tgtcccgatt & 6.39 & -88 & 3.386 & s. m. \\
\hline SCO3947 & SLI4195 & SGR3644 & $c y d C D$ & catcccgccg & 5.08 & -30 & 2.653 & s. m. \\
\hline SCO3971 & SLI4220 & SGR3620 & & tggecggtac & 7.78 & -465 & 1.631 & u. f. \\
\hline SCO4215 & SLI4452 & - & $x \ln R$ & gatgaggecg & 3.74 & -294 & 1.964 & r. \\
\hline
\end{tabular}


Table 3 Genes putatively directly regulated by S. lividans AdpA in liquid rich medium ${ }^{\text {a }}$ (Continued)

\begin{tabular}{|c|c|c|c|c|c|c|c|c|}
\hline SCO5240 & SLI5531 & SGR2274 & wble & tgtcccgatc & 5.99 & -170 & 2.246 & u. $f$. \\
\hline SCO5862 & SLI6134 & SGR1670 & cutR & tggccgaaaa & 7.69 & -99 & 1.927 & r. \\
\hline SCO6009 & SLI6398 & SGR1498 & & cttccagcca & 6.53 & -52 & 1.736 & c. p. \\
\hline
\end{tabular}

${ }^{a}$ Orthologs of S. lividans AdpA-dependent genes (listed in Additional file 2: Table S2) were analysed in silico using the S. coelicolor genome database (version 1.2.3.0 of PREDetector software [39]). AdpA-binding sites upstream from S. coelicolor genes were identified and are presented in Additional file 5: Table S4. Table 3 presents a selected subset of this complete compilation.

${ }^{\mathrm{b}}$ Gene names for S. griseus (SGR) and annotated function are from the StrepDB database [7]. Ortholog gene names were identified using StrepDB. Genes identified in other Streptomyces as being directly AdpA-regulated are in bold, and those described as being AdpA-dependent are italicized [12-15,22]. ${ }^{*}$ Binding sites in the promoters of these genes were identified in silico [22]. The SCO2921-ortholog was not annotated as a S. lividans CDS; however, our microarray data suggest that this CDS exists.

'cis-element, score, and binding site position as determined by analysing S. coelicolor genes with PREDetector [39]. When more than one putative AdpA-binding site was detected, only the one with the highest score was shown here. Other genes putatively directly regulated by S. lividans AdpA are listed in Additional file 5: Table S4. \# site found in the SCO3122 CDS at position 1447 (total gene length $1449 \mathrm{nt}$ ).

${ }^{\mathrm{d}}$ Fold change $(\mathrm{Fc})$ in gene expression in S. lividans adpA mutant relative to the parental strain with $P$-value $<0.05$, as determined by Student's $t$-test applying the Benjamini and Hochberg multiple testing correction (details in Additional file 2: Table S2).

eFrom a protein classification scheme for the S. coelicolor genome available on the Welcome Trust Sanger Institute database [37]: unknown function (u. f.), cell process (c. p.), macromolecule metabolism (m. m.), small molecule metabolism (s. m.), cell envelope (c. e.), extrachromosomal (e.), regulation (r.) and not classified (n. c.).

lividans, hyaS affects hypha aggregation and the amount of mycelium-associated undecylprodigiosin [44]; its function in S. griseus is unknown. The expression of all of bldN, SLI6392, SLI1868 and the SCO2921 ortholog (gene detected in S. lividans genome but not named in StrepDB [7]) is influenced by adpA deletion in S. lividans. It remains to be determined whether AdpA directly controls $S$. lividans adpA and bldA as described in S. coelicolor and griseus [16,23].

S. coelicolor adpA is one of 145 identified TTAcontaining genes; the production of the proteins encoded by these genes is dependent on bldA, encoding the only tRNA for the rare leucine codon TTA [46]. Our study has revealed that expression of 11 TTA-containing genes and of 24 genes regulated by $S$. coelicolor bldA $[42,47,48]$ was affected by $a d p A$ deletion in S. lividans (Additional files 4: Table S3). We show that $\operatorname{cch} A, \operatorname{cchB}$, stil, hyaS, SLI6586 and SLI6587, previously identified in S. coelicolor as bldAdependent genes, are direct targets of S. lividans AdpA [25]. Of the 29 other bldA-dependent genes, 19 are probable direct S. lividans AdpA targets: in silico analysis indicated the presence of putative AdpA-binding sites upstream from these genes (most of them with score above 4, see Additional file 5: Table S4). By analogy, this suggests that the deregulation of certain genes observed in the S. coelicolor bldA mutant may have been the consequence of S. coelicolor AdpA down-regulation, as previously suggested [49].

To predict probable direct targets of AdpA in S. lividans and contribute to knowledge of the AdpA regulon, we carried out in silico analysis of the entire S. coelicolor genome using PREDetector [39], and also restricted to the S. lividans genes identified as being AdpA-dependent (see Additional file 5: Table S4 and Table 3). We identified 95 genes probably directly activated by $S$. lividans AdpA and 67 genes that could be directly repressed (Additional file 5 : Table S4). Most of the putative AdpA-binding sites identified by this analysis are coherent with the findings of Yao et al., demonstrating the importance of $\mathrm{G}$ and $\mathrm{C}$ nucleotides at positions 2 and 4, respectively [50]. Six genes have been identified as directly regulated by AdpA in other species (adpA, bldN, wblA, SLI6392, SCO2921 orthologs, and $g l p Q 1$, as indicated in Table 3 in bold) $[10,12,15,16,18]$, and 27 more in S. griseus are also probable AdpA-direct targets (e.g. $\operatorname{cch} B$, SLI0755-0754 operon, rarA operon, scoF4, groEL1, SLI6587, SLI4345, cydAB, and ectABD, as indicated in Table 3 and Additional file 2: Table S2, underlined) $[7,12-14]$. Sixty-three of the 162 probable direct targets of AdpA in S. lividans have no ortholog in the $S$. griseus genome (Additional file 5: Table S4).

\section{Conclusions}

In conclusion, this study has extended our knowledge of the S. lividans AdpA regulon. We identified S. lividans AdpA-regulated genes by transcriptomic analysis, and used in silico analysis to identify over a hundred probable direct targets of AdpA in S. lividans. Most of them are absent from the current predicted S. griseus AdpA regulon. Discovering new $S$. lividans genes directly regulated by AdpA and that are involved in primary and secondary metabolism will provide valuable information about Streptomyces development and differentiation in liquid culture.

\section{Availability of supporting data}

Microarray data are available in the ArrayExpress database $[51,52]$ under accession number A-MEXP-2383.

\section{Additional files}

Additional file 1: Table S1. Oligonucleotides used in this study.

Additional file 2: Table S2. Complete set of genes differentially expressed in the S. lividans adpA mutant. S. coelicolor microarrays were used to test for genes differentially expressed in the S. lividans adpA mutant and wild-type 1326, at growth time point T, in liquid YEME medium. Annotated function, Fc, $P$-values, and classification of the proteins are presented according to the microarray SCO genes, by increasing SCO gene number. 
Additional file 3: Figure S1. Effect of the mutation of one AdpA-binding site in the S. lividans hyaS promoter on AdpA-binding specificity. Mutation of an AdpA-binding site in the $S$. lividans hyaS promoter region prevents formation of an AdpA-DNA complex in vitro. Sequence of the mutated AdpA-binding site (at -129 nt) and EMSA performed with the mutated hyaS promoter region are shown.

Additional file 4: Table S3. Comparison of gene expression profiles between S. coelicolor bldA-dependent and S. lividans AdpA-dependent genes. Comparison of the gene expression profiles of some S. coelicolor bldA-dependent genes whose $S$. lividans orthologs are AdpA-dependent (see Additional file 2: Table S2). Putative AdpA-binding sites were identified in silico (see Additional file 5: Table S4), suggesting that in the S. coelicolor bldA mutant, the adpA translation defect leads to bldA-dependence of the genes identified previously $[42,47,48]$.

Additional file 5: Table S4. Putative $S$. coelicolor AdpA-binding sites upstream from the $S$. lividans AdpA-dependent genes. We identified putative AdpA-binding sites in silico using the S. coelicolor genome and we analysed orthologs of S. lividans AdpA-dependent genes (based on our microarray data); the sequences and positions of the sites with the highest scores according to PREDetector are shown. S. coelicolor, S. lividans and S. griseus ortholog genes are indicated and previously identified direct or probably direct $S$. griseus AdpA-dependent genes are highlighted.

\section{Abbreviations}

qRT-PCR: Quantitative real-time PCR; ORF: Open reading frame; Fc: Fold change; $C_{T}$ : Critical threshold cycle; BLAST: Basic local alignment search tool; EMSA: Electrophoretic mobility shift assay; AdpA-His6: Recombinant AdpA protein with a six-histine tag at the C-terminus; CDS: Coding DNA sequence; CS: Coding strand; NCS: Non coding strand; u. f.: Unknown function; c. p.: Cell process; $\mathrm{m}$. m.: Macromolecule metabolism; s. m.: Small molecule metabolism; c. e.: Cell envelope; e.: Extrachromosomal; r.: Regulation; n. c.: Not classified.

\section{Competing interests}

The authors declare that they have no competing interests.

\section{Authors' contributions}

AG, NB and PM wrote and revised the manuscript. CP and JYC have given final approval for this version to be published. PM helped AG to design the project. AG performed GRT-PCR, EMSA and in silico analysis; and prepared Figures, Tables and Additional files. NB purified AdpA-His ${ }_{6}$ protein. CP carried out the microarray experiments. JYC helped CP with the statistical analysis of microarray results and wrote the associated Methods sections. AG interpreted the microarrays data. MG help with qRT-PCR experiments and provided technical support. All authors read and approved the final manuscript.

\section{Authors' information}

AG performed GRT-PCR and EMSA experiments while working at Pasteur Institute. Her current address is Centre for Bacterial Cell Biology, Institute for Cell and Molecular Biosciences, Newcastle University, Newcastle-upon-Tyne NE2 4HH, UK.

\section{Acknowledgements}

We thank T. Msadek, S. Dubrac, E. Johnson and J.-L. Pernodet for helpful discussion and critical reading of the manuscript, and O. Poupel for assistance with qRT-PCR analysis. We are grateful to G. Bucca for her advice and help with microarray handling. We thank Alex Edelman \& Associates for correcting the manuscript.

This work was supported by research funds from the Institut Pasteur and Centre National de Recherche Scientifique. A. Guyet was the recipient of fellowships from the Ministère de l'Education Nationale, de la Recherche et de la Technologie, the Pasteur-Weizmann foundation and the ERA-IB European grant. AG thanks BBSRC and R. Daniel for his constant support during the preparation of this manuscript.

\section{Author details}

${ }^{1}$ Unité de Biologie des Bactéries Pathogènes à Gram-Positif, Institut Pasteur, CNRS URA 2172, 28 rue du Docteur Roux, 75724 Paris Cedex 15, France. 'Unité Biologie des Spirochètes, Institut Pasteur, 28 rue du Docteur Roux, 75724 Paris Cedex 15, France. ${ }^{3}$ Plateforme Transcriptome et Epigenome (PF2), 28 rue du Docteur Roux, 75724 Paris Cedex 15, France.
Received: 8 November 2013 Accepted: 27 March 2014

Published: 3 April 2014

\section{References}

1. Elliot MA, Buttner MJ, Nodwell JR: Multicellular development in Streptomyces. In Myxobacteria: Multicellularity and Differentiation. Edited by Whitworth DE. Washington, D. C: ASM Press; 2008:419-438.

2. Manteca A, Alvarez R, Salazar N, Yague P, Sanchez J: Mycelium differentiation and antibiotic production in submerged cultures of Streptomyces coelicolor. Appl Environ Microbiol 2008, 74(12):3877-3886.

3. Ohnishi Y, Kameyama S, Onaka H, Horinouchi S: The A-factor regulatory cascade leading to streptomycin biosynthesis in Streptomyces griseus: identification of a target gene of the A-factor receptor. Mol Microbiol 1999, 34(1):102-111.

4. Takano E, Tao M, Long F, Bibb MJ, Wang L, Li W, Buttner MJ, Bibb MJ, Deng ZX, Chater KF: A rare leucine codon in adpA is implicated in the morphological defect of bldA mutants of Streptomyces coelicolor. Mol Microbiol 2003, 50(2):475-486.

5. Nguyen KT, Tenor J, Stettler H, Nguyen LT, Nguyen LD, Thompson CJ: Colonial differentiation in Streptomyces coelicolor depends on translation of a specific codon within the adpA gene. J Bacterio/ 2003, 185(24):7291-7296.

6. McCormick JR, Flardh K: Signals and regulators that govern Streptomyces development. FEMS Microbiol Rev 2012, 36(1):206-231.

7. StrepDB -The Streptomyces annotation server. http://strepdb.streptomyces. org.uk/.

8. Gallegos MT, Schleif R, Bairoch A, Hofmann K, Ramos JL: AraC/XylS family of transcriptional regulators. Microbiol Mol Biol Rev 1997, 61(4):393-410.

9. Egan SM: Growing repertoire of AraC/XylS activators. J Bacteriol 2002, 184(20):5529-5532

10. Yamazaki H, Tomono A, Ohnishi Y, Horinouchi S: DNA-binding specificity of AdpA, a transcriptional activator in the A-factor regulatory cascade in Streptomyces griseus. Mol Microbiol 2004, 53(2):555-572.

11. Horinouchi S: Mining and polishing of the treasure trove in the bacterial genus Streptomyces. Biosci Biotechnol Biochem 2007, 71(2):283-299.

12. Akanuma G, Hara H, Ohnishi Y, Horinouchi S: Dynamic changes in the extracellular proteome caused by absence of a pleiotropic regulator AdpA in Streptomyces griseus. Mol Microbiol 2009, 73(5):898-912.

13. Hara $\mathrm{H}$, Ohnishi $Y$, Horinouchi S: DNA microarray analysis of global gene regulation by A-factor in Streptomyces griseus. Microbiology 2009, 155(Pt 7):2197-2210.

14. Higo A, Hara H, Horinouchi S, Ohnishi Y: Genome-wide distribution of AdpA, a global regulator for secondary metabolism and morphological differentiation in Streptomyces, revealed the extent and complexity of the AdpA regulatory network. DNA Res 2012, 19(3):259-274.

15. Lee HN, Kim JS, Kim P, Lee HS, Kim ES: Repression of antibiotic downregulator WbIA by AdpA in Streptomyces coelicolor. Appl Environ Microbiol 2013, 79(13):4159-4163.

16. Wolanski M, Donczew R, Kois-Ostrowska A, Masiewicz P, Jakimowicz D, Zakrzewska-Czerwinska J: The level of AdpA directly affects expression of developmental genes in Streptomyces coelicolor. J Bacteriol 2011, 193(22):6358-6365.

17. Liu G, Chater KF, Chandra G, Niu G, Tan H: Molecular regulation of antibiotic biosynthesis in Streptomyces. Microbiol Mol Biol Rev 2013, 77(1):112-143.

18. Kato J, Ohnish Y, Horinouchi S: Autorepression of AdpA of the AraC/XylS family, a key transcriptional activator in the A-factor regulatory cascade in Streptomyces griseus. J Mol Biol 2005, 350(1):12-26.

19. Ohnishi Y, Yamazaki H, Kato JY, Tomono A, Horinouchi S: AdpA, a central transcriptional regulator in the A-factor regulatory cascade that leads to morphological development and secondary metabolism in Streptomyces griseus. Biosci Biotechnol Biochem 2005, 69(3):431-439.

20. Xu D, Kim TJ, Park ZY, Lee SK, Yang SH, Kwon HJ, Suh JW: A DNA-binding factor, ArfA, interacts with the bldH promoter and affects undecylprodigiosin production in Streptomyces lividans. Biochem Biophys Res Commun 2009, 379(2):319-323.

21. den Hengst CD, Tran NT, Bibb MJ, Chandra G, Leskiw BK, Buttner MJ: Genes essential for morphological development and antibiotic production in Streptomyces coelicolor are targets of BldD during vegetative growth. Mol Microbiol 2010, 78(2):361-379.

22. Xu W, Huang J, Lin R, Shi J, Cohen SN: Regulation of morphological differentiation in S. coelicolor by RNase III (AbsB) cleavage of mRNA encoding the AdpA transcription factor. Mol Microbiol 2010, 75(3):781-791. 
23. Higo A, Horinouchi S, Ohnishi Y: Strict regulation of morphological differentiation and secondary metabolism by a positive feedback loop between two global regulators AdpA and BldA in Streptomyces griseus. Mol Microbiol 2011, 81(6):1607-1622.

24. Cruz-Morales P, Vijgenboom E, Iruegas-Bocardo F, Girard G, Yanez-Guerra LA, Ramos-Aboites HE, Pernodet JL, Anne J, van Wezel GP, Barona-Gomez F: The genome sequence of Streptomyces lividans 66 reveals a novel tRNAdependent peptide biosynthetic system within a metal-related genomic island. Genome Biol Evol 2013, 5(6):1165-1175.

25. Guyet A, Gominet M, Benaroudj N, Mazodier P: Regulation of the clpP1clpP2 operon by the pleiotropic regulator AdpA in Streptomyces lividans. Arch Microbiol 2013, 195(12):831-841.

26. Murakami T, Holt TG, Thompson CJ: Thiostrepton-induced gene expression in Streptomyces lividans. J Bacteriol 1989, 171(3):1459-1466.

27. Kieser T, Bibb MJ, Buttner MJ, Chater KF, Hopwood DA: Practical Streptomyces genetics. Norwich: John Innes Foundation; 2000.

28. Surrey University Streptomyces coelicolor microarray resource. http://www.surrey.ac.uk/fhms/microarrays/.

29. Bucca G, Brassington AM, Hotchkiss G, Mersinias V, Smith CP: Negative feedback regulation of $d n a K, c l p B$ and lon expression by the DnaK chaperone machine in Streptomyces coelicolor, identified by transcriptome and in vivo DnaK-depletion analysis. Mol Microbiol 2003, 50(1):153-166.

30. Bellier A, Mazodier P: ClgR, a novel regulator of $c l p$ and lon expression in Streptomyces. J Bacteriol 2004, 186(10):3238-3248.

31. Ralph SA, Bischoff E, Mattei D, Sismeiro O, Dillies MA, Guigon G, Coppee JY, David PH, Scherf A: Transcriptome analysis of antigenic variation in Plasmodium falciparum - var silencing is not dependent on antisense RNA. Genome Biol 2005, 6(11):R93.

32. R Development Core Team: R: A language and environment for statistical computing. http://www.R-project.org.

33. Yang YH, Dudoit S, Luu P, Lin DM, Peng V, Ngai J, Speed TP: Normalization for cDNA microarray data: a robust composite method addressing single and multiple slide systematic variation. Nucleic Acids Res 2002, 30(4):e15.

34. marray - a Bioconductor package for exploratory analysis for two-color spotted microarray data. http://www.bioconductor.org/packages/release/ bioc/html/marray.html.

35. Reiner A, Yekutieli D, Benjamini Y: Identifying differentially expressed genes using false discovery rate controlling procedures. Bioinformatics 2003, 19(3):368-375.

36. Delmar P, Robin S, Daudin JJ: VarMixt: efficient variance modelling for the differential analysis of replicated gene expression data. Bioinformatics 2005, 21(4):502-508.

37. The Sanger Institute Streptomyces coelicolor protein classification scheme. ftp://ftp.sanger.ac.uk/pub/S_coelicolor/classwise.txt.

38. Livak KJ, Schmittgen TD: Analysis of relative gene expression data using real-time quantitative PCR and the $2^{-\Delta \Delta C_{T}}$ method. Methods 2001, 25(4):402-408.

39. Hiard S, Maree R, Colson S, Hoskisson PA, Titgemeyer F, van Wezel GP, Joris B, Wehenkel L, Rigali S: PREDetector: a new tool to identify regulatory elements in bacterial genomes. Biochem Biophys Res Commun 2007, 357(4):861-864.

40. Derre I, Rapoport G, Msadek T: CtsR, a novel regulator of stress and heat shock response, controls $c / p$ and molecular chaperone gene expression in Gram-positive bacteria. Mol Microbiol 1999, 31(1):117-131.

41. Jayapal KP, Lian W, Glod F, Sherman DH, Hu WS: Comparative genomic hybridizations reveal absence of large Streptomyces coelicolor genomic islands in Streptomyces lividans. BMC Genomics 2007, 8:229.

42. Hesketh A, Bucca G, Laing E, Flett F, Hotchkiss G, Smith CP, Chater KF: New pleiotropic effects of eliminating a rare tRNA from Streptomyces coelicolor, revealed by combined proteomic and transcriptomic analysis of liquid cultures. BMC Genomics 2007, 8:261.

43. Lautru S, Deeth RJ, Bailey LM, Challis GL: Discovery of a new peptide natural product by Streptomyces coelicolor genome mining. Nat Chem Biol 2005, 1(5):265-269.

44. Koebsch I, Overbeck J, Piepmeyer S, Meschke H, Schrempf H: A molecular key for building hyphae aggregates: the role of the newly identified Streptomyces protein HyaS. Microb Biotechnol 2009, 2(3):343-360.

45. Chun YJ, Shimada T, Sanchez-Ponce R, Martin MV, Lei L, Zhao B, Kelly SL, Waterman MR, Lamb DC, Guengerich FP: Electron transport pathway for a Streptomyces cytochrome P450: cytochrome P450 105D5-catalyzed fatty acid hydroxylation in Streptomyces coelicolor A3(2). J Biol Chem 2007, 282(24):17486-17500.

46. Li WC, Wu J, Tao WX, Zhao CH, Wang YM, He XY, Chandra G, Zhou XF, Deng ZX, Chater KF, Tao MF: A genetic and bioinformatic analysis of Streptomyces coelicolor genes containing TTA codons, possible targets for regulation by a developmentally significant tRNA. FEMS Microbiol Lett 2007, 266(1):20-28

47. Kim DW, Chater K, Lee KJ, Hesketh A: Changes in the extracellular proteome caused by the absence of the bldA gene product, a developmentally significant tRNA, reveal a new target for the pleiotropic regulator AdpA in Streptomyces coelicolor. J Bacteriol 2005, 187(9):2957-2966.

48. Kim DW, Chater KF, Lee KJ, Hesketh A: Effects of growth phase and the developmentally significant bldA-specified tRNA on the membraneassociated proteome of Streptomyces coelicolor. Microbiol Sgm 2005, 151:2707-2720.

49. Chater KF, Chandra G: The use of the rare UUA codon to define "Expression Space" for genes involved in secondary metabolism, development and environmental adaptation in Streptomyces. J Microbio/ 2008, 46(1):1-11.

50. Yao MD, Ohtsuka J, Nagata K, Miyazono Kl, Zhi Y, Ohnishi Y, Tanokura M: Complex structure of the DNA-binding domain of AdpA, the global transcription factor in Streptomyces griseus, and a target duplex DNA reveals the structural basis of its tolerant DNA sequence specificity. J Biol Chem 2013, 288(43):31019-31029.

51. ArrayExpress database. http://www.ebi.ac.uk/arrayexpress/.

52. Rustici G, Kolesnikov N, Brandizi M, Burdett T, Dylag M, Emam I, Farne A, Hastings E, Ison J, Keays M, Kurbatova N, Malone J, Mani R, Mupo A, Pedro Pereira R, Pilicheva E, Rung J, Sharma A, Tang YA, Ternent T, Tikhonov A, Welter D, Williams E, Brazma A, Parkinson H, Sarkans U: ArrayExpress updatetrends in database growth and links to data analysis tools. Nucleic Acids Res 2013, 41(Database issue):D987-D990

doi:10.1186/1471-2180-14-81

Cite this article as: Guyet et al:: Identified members of the Streptomyces lividans AdpA regulon involved in differentiation and secondary metabolism. BMC Microbiology 2014 14:81.

\section{Submit your next manuscript to BioMed Central and take full advantage of:}

- Convenient online submission

- Thorough peer review

- No space constraints or color figure charges

- Immediate publication on acceptance

- Inclusion in PubMed, CAS, Scopus and Google Scholar

- Research which is freely available for redistribution

Submit your manuscript at www.biomedcentral.com/submit
C BioMed Central 